\title{
Multiscale Computational Modeling of Deformation Mechanics and Intergranular Fracture in Nanocrystalline Copper
}

\author{
V. Péron-Lührs ${ }^{\mathrm{a}}$, F. Sansoz ${ }^{\mathrm{b}}$, A. Jérusalem ${ }^{\mathrm{c}}$, L. Noels $^{\mathrm{a}, *}$ \\ ${ }^{a}$ Department of Aerospace $\&$ Mechanical Engineering - Computational $\&$ Multiscale Mechanics of Materials, University of \\ Liège, Chemin des Chevreuils 1, B4000 Liège Belgium \\ ${ }^{b}$ Mechanical engineering and materials science programs, School of Engineering, The University of Vermont, Burlington, VT \\ 05405, USA \\ ${ }^{c}$ Department of Engineering Science, Parks Road, University of Oxford, OX1 3PJ, UK
}

\begin{abstract}
This study presents the development and validation of a two-scale numerical method aimed at predicting the mechanical behavior and the inter-granular fracture of nanocrystalline (NC) metals under deformation. The material description is based on two constitutive elements, the grains (or bulk crystals) and the grainboundaries (GBs), both having their behavior determined atomistically using the quasicontinuum (QC) method by simulating the plastic deformation of [1ํㅣ] tilt crystalline interfaces undergoing simple shear, tension and nano-indentation. Unlike our previous work [V. Péron-Lührs et al., JMPS, 2013] however, the GB thickness is here calibrated in the model, providing more accurate insight into the GB widths according to the interface misorientation angle. In this contribution, the new two-scale model is also validated against fullyatomistic NC simulations tests for two low-angle and high-angle textures and two grain sizes. A simplified strategy aimed at predicting the mechanical behavior of more general textures without the need to run more QC simulations is also proposed, demonstrating significant reduction in computational cost compared to full atomistic simulations. Finally, by studying the response of dogbone samples made of NC copper, we show in this paper that such a two-scale model is able to quantitatively capture the differences in mechanical behavior of NC metals as a function of the texture and grain size, as well as to accurately predict the processes of inter-granular fracture for different GB character distributions. This two-scale method is found to be an effective alternative to other atomistic methods for the prediction of plasticity and fracture in NC materials with a substantial number of 2-D grains such as columnar-grained thin films for micro-scale electro-mechanical devices.
\end{abstract}

Keywords: Nanocrystalline metals, Finite element model, Quasicontinuum method, two-scale model, Grain-boundary 


\section{Introduction}

3

Nanocrystalline (NC) materials are known to possess remarkable physical and mechanical properties such as ultrahigh strength compared with their coarse-grained counterparts $[1,2,3,4,5,6,7,8]$. At this length scale, the plastic deformation is considered to change from intragranular to intergranular by a mechanism in which the grain boundary (GB) character distribution (GBCD) is promoted and controls the NC mechanical behavior [9]. This transition from intragranular to intergranular or GB-mediated plasticity is assumed to control the ductility and fracture behavior of NC materials. However, understanding the evolution of fracture in NC materials is at its infancy experimentally, whereas a failure model at the atomic level is still unavailable. This work aims to develop an atomistically-informed multiscale model for the quantitative prediction of the fracture behavior in NC solids.

Molecular dynamics (MD) simulations have already revealed unusual mechanisms at low temperatures, such as GB sliding and intragranular slip involving dislocation emissions and absorptions at GBs [3, 10, 11, $12,13,14]$, but suffer from the requirement to consider the dynamics of all atoms, thus imposing drastic limitations on the size of the simulated sample. On the other hand, continuum models, which do not suffer from such limitation, have been mainly limited to the description of grain size dependency [15], strain localization $[16,17]$ and failure processes $[15,18]$. It appears however that none of these models can predict the plastic deformation of relatively large NC structures while retaining sufficient precision to account for the mechanisms involved at the nanoscale.

In a recent work [19], an original numerical multiscale approach was proposed to gain predictive understanding of the mechanical behavior of NC metals as a function of their GBCD. This model assumes that GBs are embedded in a continuum matrix and incorporate full GB elasto-plastic constitutive laws determined by atomistic simulations, thereby paving the way to simulate and characterize intergranular fracture in NC materials without the need to fully model all GBs atomistically. In this two-scale framework, the material description was based on the mechanical behavior of two constitutive elements, namely, bulk crystals (or grains) and GBs. The constitutive laws for these two constitutive elements were calibrated atomistically using the quasicontinuum (QC) method [20, 21]. An explicit FCC crystal plasticity constitutive model [22] was used for the grains. This formulation improves the original implicit formulation of the forest dislocation hardening model proposed in Ref. [23] and enables large scale computations. The crystal plasticity model was previously characterized using nanoindentation QC simulations [19]. GBs were treated as surfaces of

\footnotetext{
*. Corresponding author, Phone: +32436648 26, Fax: +3243669505
}

Email address: L.Noels@ulg.ac.be (L. Noels) 
discontinuities with a finite thickness embedded in the continuum. In Ref. [19], parameters of the GBs constitutive model, including plastic and damage responses, were calibrated using the QC method by means of tensile and shear tests, following Ref. [24, 25], so as to account for both GB sliding and GB opening modes. However, in that work, the thickness of the GBs was arbitrarily taken as $1 \mathrm{~nm}$ [19]. The model is improved here by calibrating the GB thickness parameter, validated against full atomistic results, and tested for intergranular fracture predictions.

Indeed, the objective of the present work is to demonstrate the ability of this two-scale model in predicting the fracture of NC metals for different textures and grain sizes. To this end, we first validate the purely continuum model by comparing it with fully-atomistic QC simulations for two NCs with two different textures, namely high-angle (HA) and low-angle (LA), and two different mean grain sizes. It is found that both models predict the same failure evolution in the GB networks for each texture and grain size. Once the validation step is over, an adequate fitting of the HABs calibration parameters according to their misorientation is proposed. This fitting step allows for the simulation of larger NC with a HA texture consisting of a substantial number of grains without having to run all HABs QC simulations. As an illustration, two NC dogbones, consisting of 103 grains and 251 GBs and presenting the same HA texture with two different mean grain sizes are subjected to tensile loading. These simulations highlight the ability of the two-scale model to predict the intergranular fracture of larger NCs than those conventionally encountered when dealing with pure atomistic simulations, while saving computational time.

The paper is organized as follows. Section 2 presents the details of the two-scale model and the constitutive laws used for grains and GBs. Section 3 is devoted to the QC calibration; GBs QC simulations and nanoindentation tests are presented with a particular focus on the GB width effect. Section 3 also presents the QC results fitting process designed to facilitate HA-type texture simulations. In Section 4, the full calibrated two-scale continuum model is compared to the fully-atomistic QC model for validation. Finally, this methodology is applied in Section 5 to HA-type NC dogbones simulations.

\section{The two-scale model}

\subsection{Constitutive framework}

In this section, the continuum framework with embedded GBs is summarized, following the study in Ref. [9]. The main equations for the bulk material are also presented, based on Ref. [22, 23, 26]. The reader is invited to refer to Ref. [19] for more details. 
a)

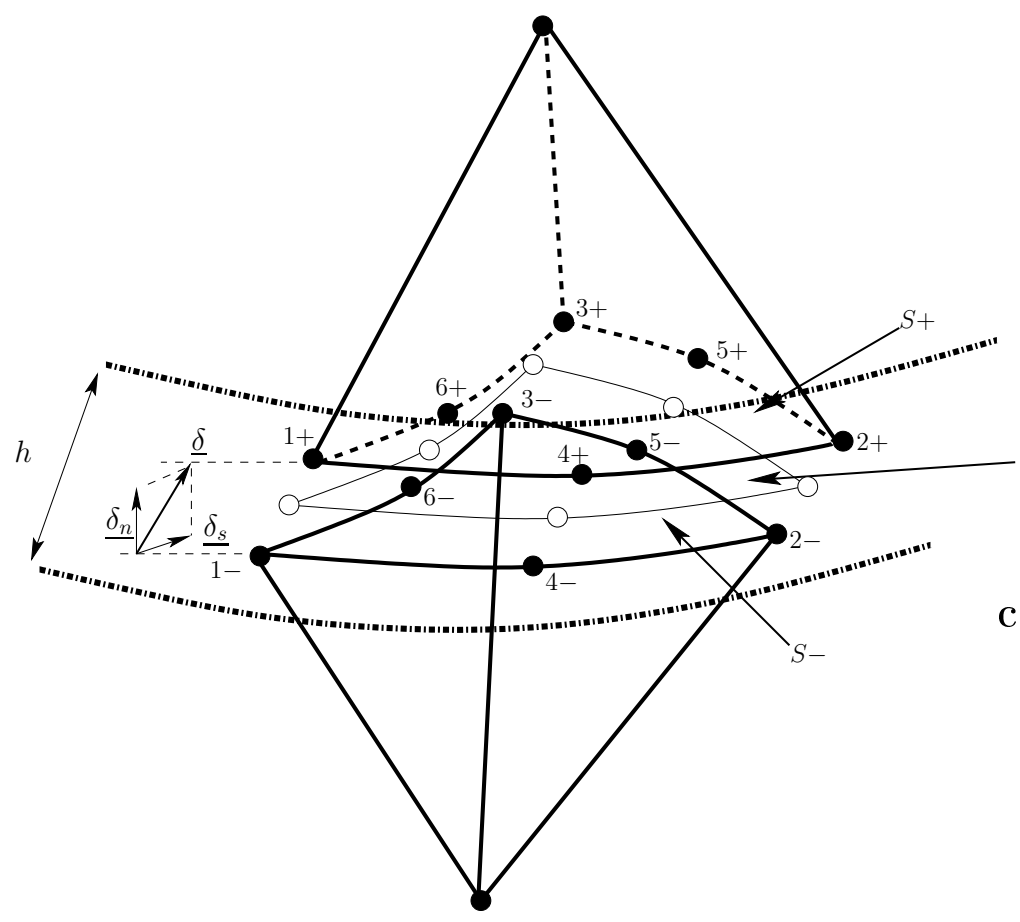

b)

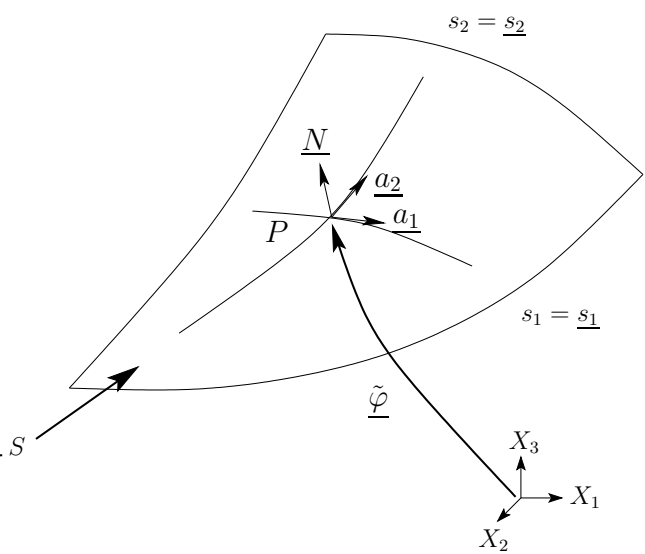

c)

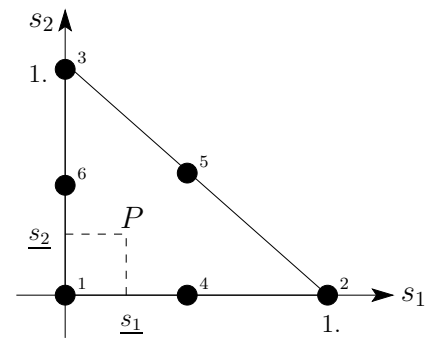

Figure 1: a) Schematics of a GB element. Two tetrahedra belonging to two adjacent crystals separated by an interface element at the GB: $S+$ and $S$ - are respectively the facets corresponding to the tetrahedra on the positive and negative sides, as defined by the positive surface normal $\underline{\boldsymbol{N}}$, and $S$ is the midsurface. The displacement jump $\underline{\boldsymbol{\delta}}$, its opening $\underline{\boldsymbol{\delta}_{\boldsymbol{n}}}$ and sliding $\underline{\boldsymbol{\delta}_{\boldsymbol{s}}}$ components, as well as the GB thickness $h$ are also indicated. b) Deformed midsurface $\mathrm{S}$ and its associated curvilinear coordinate system as well as the representation of the mean deformation mapping $\underline{\tilde{\varphi}}$. c) Standard element configuration and natural coordinate system.

\subsubsection{Grain-boundary constitutive model}

The kinematics of the deformation mapping of a GB was developed by considering the GB as a surface discontinuity embedded in the finite element discretization, and was based on the relative motion of the two surfaces $S+$ and $S$-, corresponding to the facets of the tetrahedra on the positive and negative sides, respectively, as shown in Figure 1a. To this end, the framework presented in Ref. [27] is used. The local stress state is described by the Cauchy stress tensor $\underline{\boldsymbol{\sigma}}$ whereas local information about the material deformation is conveyed by the deformation gradient field $\underline{\underline{\epsilon}}$. The material models required to evaluate $\underline{\underline{\boldsymbol{\sigma}}}$ in the bulk as well as the surface traction $\underline{\bar{t}}$ at the GBs are defined below. The mean deformation mapping is defined as set in Ref. [27]

$$
\underline{\tilde{\varphi}}=\frac{1}{2}\left(\underline{\varphi}^{+}+\underline{\varphi}^{-}\right)
$$

In Equation (1), $\underline{\tilde{\varphi}}$ is the deformation of the midsurface $S$, and $\underline{\varphi}^{+}$and $\underline{\varphi}^{-}$are the deformation mappings of the surfaces $S+$ and $S$-, respectively. By using Equation (1) we recover the original deformation mapping 
on both sides of the GB

$$
\underline{\varphi}^{ \pm}=\underline{\tilde{\varphi}} \pm \frac{1}{2}\left(\underline{\varphi}^{+}-\underline{\varphi}^{-}\right)=\underline{\tilde{\varphi}} \pm \frac{1}{2} \underline{\delta}
$$

71 where

$$
\underline{\delta}=\llbracket \underline{\varphi} \rrbracket=\underline{\varphi}^{+}-\underline{\varphi}^{-}
$$

$$
\underline{\underline{\boldsymbol{\epsilon}}}=\underbrace{\frac{\underline{\boldsymbol{\delta}}_{n} \cdot \underline{\boldsymbol{N}}_{3}}{h} \underline{\boldsymbol{N}}_{3} \otimes \underline{\boldsymbol{N}}_{3}}_{\underline{\underline{\boldsymbol{\epsilon}_{n}}}}+\underbrace{\frac{\underline{\boldsymbol{\delta}}_{s} \cdot \underline{\boldsymbol{N}_{1}}}{h} \frac{1}{2}\left(\underline{\boldsymbol{N}}_{1} \otimes \underline{\boldsymbol{N}}_{3}+\underline{\boldsymbol{N}}_{3} \otimes \underline{\boldsymbol{N}}_{1}\right)+\frac{\underline{\boldsymbol{\delta}}_{s} \cdot \underline{\boldsymbol{N}}_{2}}{h} \frac{1}{2}\left(\underline{\boldsymbol{N}}_{2} \otimes \underline{\boldsymbol{N}}_{3}+\underline{\boldsymbol{N}}_{3} \otimes \underline{\boldsymbol{N}}_{2}\right)}_{\underline{\underline{\boldsymbol{\epsilon}_{s}}}}
$$

The displacement jumps can be decomposed into a GB opening vector and a sliding vector as follows

$$
\underline{\boldsymbol{\delta}}_{n}=(\underline{\boldsymbol{\delta}} \cdot \underline{\boldsymbol{N}}) \underline{\boldsymbol{N}}=(\underline{\boldsymbol{N}} \otimes \underline{\boldsymbol{N}}) \cdot \underline{\boldsymbol{\delta}}
$$

$$
\underline{\boldsymbol{\delta}}_{s}=\underline{\boldsymbol{\delta}}-\underline{\boldsymbol{\delta}}_{n}=(\underline{\underline{\boldsymbol{I}}}-\underline{\boldsymbol{N}} \otimes \underline{\boldsymbol{N}}) \cdot \underline{\boldsymbol{\delta}}
$$

It is assumed that this kinematics imposes a constant deformation gradient across the thickness $h$ of the GB. This assumption is justified by the low number of atoms within the GB. This gradient of deformation can be expressed in the local orthonormal reference frame

$$
\left(\underline{\boldsymbol{N}}_{1}, \underline{\boldsymbol{N}}_{2}, \underline{\boldsymbol{N}}_{3}\right)=\left(\left(\underline{\boldsymbol{a}}_{1} /\left|\underline{\boldsymbol{a}}_{1}\right|\right),\left(\underline{\boldsymbol{N}} \times \underline{\boldsymbol{a}}_{1} /\left|\underline{\boldsymbol{N}} \times \underline{\boldsymbol{a}}_{1}\right|\right), \underline{\boldsymbol{N}}\right)
$$

as

84 From Equation (8), $\underline{\underline{\boldsymbol{\epsilon}}}$ is the sum of two quantities; a normal opening part $\underline{\underline{\boldsymbol{\epsilon}_{\boldsymbol{n}}}}$ and a sliding part $\underline{\underline{\boldsymbol{\epsilon}_{\boldsymbol{s}}}}$. In [19], $h$, naturally defining a characteristic length scale of GBs in the model, was set to $1 \mathrm{~nm}$ following past works $[28,29]$. However, assigning a fixed value to $h$ does not account for the different thicknesses between each 

as a parameter obtained from the calibration process. The traction is eventually expressed as

$$
\overline{\bar{t}}=h \underline{\underline{\sigma}}: \frac{\partial \underline{\underline{\epsilon}}}{\partial \underline{\underline{\delta}}}=\underline{\underline{\sigma}} \cdot \underline{\boldsymbol{N}}_{3}
$$

Here, only the sliding component undergoes plastic deformations. In fact, when running GB opening simulations with QC, only an elastic behavior, without plasticity, was observed until decohesion progressively occurs [19]. This most likely arises from the perfect nature of the GB simulated with QC. Thus using a damage law appears to be the best way to approximate the opening behavior of GBs. Therefore a damage parameter $D$ is included in the GB opening mechanical behavior. The elasto-plastic model described in Ref. [9] is used to compute the sliding part $\underline{\underline{\sigma}}^{s l}$ of the effective stress tensor and is characterized by the yield stress tensor $\sigma_{p}$ with

$$
\sigma_{p}=\sigma_{0}\left(1+\frac{\overline{\epsilon_{p}}}{\epsilon_{0}}\right)
$$

where $\bar{\epsilon}_{p}$ is the equivalent plastic strain, $\sigma_{0}$ is the initial yield stress, and $\epsilon_{0}$ is the reference plastic strain. The damage parameter $D$ is evaluated from the normal opening $\underline{\boldsymbol{\delta}}_{n} \cdot \underline{\boldsymbol{N}}$. While this opening remains relatively small, the opening stress ${\underline{\underline{\boldsymbol{\sigma}^{p}}}}^{\boldsymbol{p}}$ remains smaller in norm than the critical stress $\sigma_{c}$ and $D=0$. Once $\sigma_{c}$ is reached, $D$ increases in an irreversible way, and eventually reaches 1 for a critical opening $\delta_{c}$. Finally the stress tensor is directly computed from

$$
\underline{\underline{\sigma}}=(1-D)\left(\underline{\underline{\bar{\sigma}}}^{s l}+\underline{\underline{\bar{\sigma}}}^{o p}\right)
$$

\subsubsection{Grain constitutive model}

We adopt the explicit formulation described in Ref. [22] for FCC polycrystalline plasticity. This formulation somehow improves the original implicit formulation of the forest dislocation hardening model proposed in Ref. [23] by facilitating large scale computations. A summary of this formulation can be found elsewhere [26] and we provide here the main equations of the model in order to highlight the relevant parameters calibrated with the QC method by nanoindentation tests.

In this framework the following power-law is used to describe the shear rate deformation of each slip 
system $\alpha$

$$
\dot{\gamma}^{\alpha}= \begin{cases}\dot{\gamma}_{0}\left[\left(\frac{\tau^{\alpha}}{g^{\alpha}}\right)^{\frac{1}{m}}-1\right], & \text { if } \tau^{\alpha} \geq 0 \\ 0, & \text { otherwise }\end{cases}
$$

where $\dot{\gamma}_{0}$ is the reference shear strain rate, $m$ the strain-rate sensitivity exponent, and where $g^{\alpha}$ and $\tau^{\alpha}$ are the critical resolved shear stress (CRSS) and the resolved shear stress on slip system $\alpha$, respectively. Based on statistical mechanics [30], the evolution of the flow stresses in the case of multiple slip systems is found to be governed by a diagonal hardening law

$$
\dot{g}^{\alpha}=\sum_{\beta} h^{\alpha \beta} \dot{\gamma}^{\beta}
$$

where $h^{\alpha \beta}$ are the diagonal hardening moduli.

Table 1 provides the constitutive model parameters used in our simulations for pure copper [22]. The remaining parameter, $g_{0}$, is the initial value for $g^{\alpha}$, and depends on the grain diameter $d$ and on the texture type (HA or LA) considered. This key value is calibrated from nanoindentation QC simulations.

\begin{tabular}{llll}
\hline Parameter & Value & Parameter & Value \\
\hline$C_{11}$ & $168.4 \mathrm{GPa}$ & $\dot{\gamma}_{0}$ & $10 s^{-1}$ \\
$C_{44}$ & $75.4 \mathrm{GPa}$ & $m$ & 0.005 \\
$C_{12}$ & $121.4 \mathrm{GPa}$ & $g_{0}$ & $f(d$, texture $)$ \\
\hline
\end{tabular}

TABLE 1: Constitutive model parameters for pure copper.

\section{Calibration with the QC method}

In this section we summarize the method used to calibrate atomistically the two-scale model aimed at simulating the NCs for two different textures or GBCDs (HA and LA). To this end, both the remaining bulk parameter $\left(g_{0}\right)$ for different grain sizes and the GB parameters $\left(\sigma_{0}, G, \sigma_{c}, \delta_{c}, h\right)$ have to be calibrated for different misorientations. We also provide the influence of the GB widths on the behavior of the representative volume element (RVE) studied. Ultimately, we expose the QC results fitting process for larger HA-type texture NC simulations.

The QC method allows for the prediction of the equilibrium configuration of a system of atoms by energy minimization, given externally imposed forces or displacements. However, all atoms are not explicitly represented and regions of small deformation gradients are treated as continuum media by use of the finite element method. This method thus enables the modeling of large-scale atomistic systems without loss of 

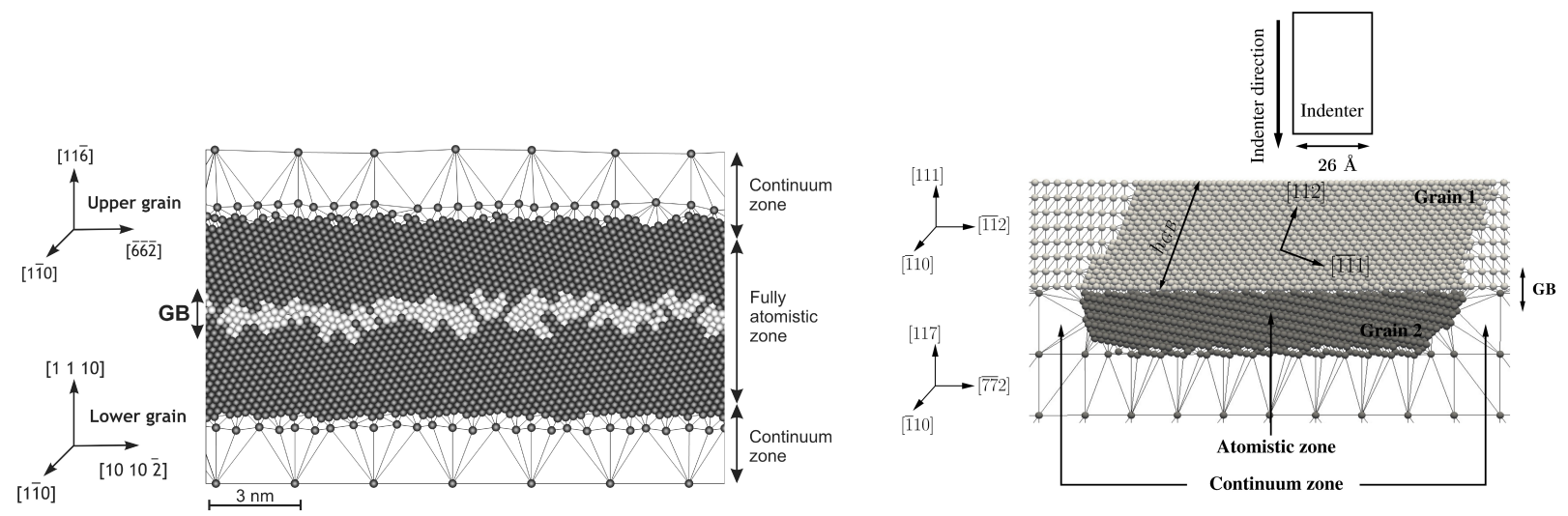

Figure 2: a) QC sliding/opening model of one HAB. b) QC nanoindentation model for one HAB with a pseudo grain size set to $h_{G B}=6.56 \mathrm{~nm}$.

accuracy in the atomistic areas while being faster than classical molecular simulations. This method was used in Ref. [19] to calibrate GB and grain mechanical behaviors.

At the nanoscale, the GB constitutive parameters were provided by simulating the GBs as done in Ref. $[24,25]$ where 2-D bicrystals were subjected to shear and tensile loading conditions in order to characterize the sliding and the opening/decohesion behavior, respectively, see Figure 2a. With the GB shearing simulations, the maximum shear strength $\sigma_{0}$ and the shear modulus $G$ were extracted from the evolution of the shear stress as a function of the applied shear strain. With regards to the GB decohesion behavior, the opening critical stress $\sigma_{c}$ as well as the critical opening $\delta_{c}$ were calibrated from stress-strain curves.

The model of nanoindentation exposed in Ref. [21, 31] was used to simulate the interaction of partial dislocation motions with GBs belonging to both HAB or LAB types. Misorientations lower than $9^{\circ}$ were considered in this work as being LABs so as to correspond to all the available LABs definitions in the literature $[32,33,34]$. We emphasize that all GBs present the same [1̄o] tilt axis. In order to take into account the grain sizes, we proposed in [19] to depart from the approach presented in Ref. [21, 31] by varying the distance $h_{G B}$ (pseudo grain size) separating the indented surface from the GB, see Figure $2 \mathrm{~b}$. For each simulation, the CRSS was extracted to enable the calibration of $g_{0}$ which is the initial value for $g^{\alpha}$ in the forest dislocation hardening model presented above. Using this calibration model enabled to capture the CRSS of slip systems $\alpha$ not only according to the GB nature (HAB or LAB) but also according to the grain size. Further information on the computational techniques used for grain and GBs calibrations is available in Ref. [19]. 


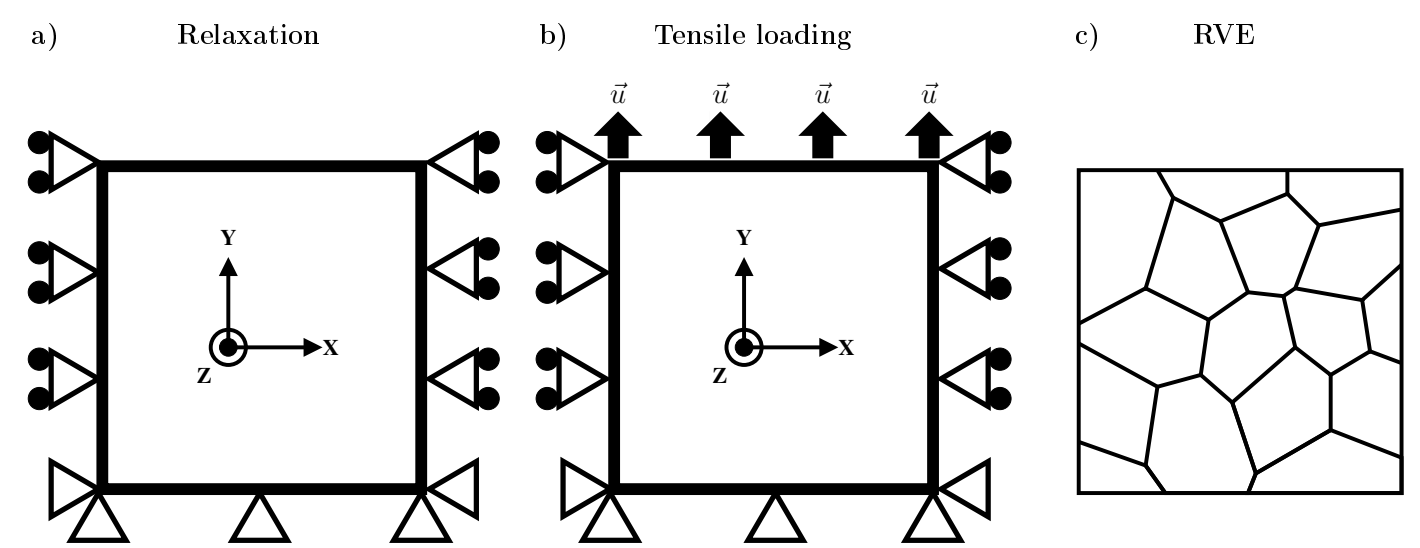

FIgURE 3: NC boundary conditions and RVE. a) Loading conditions, used for both models. b) Relaxation step, only for fully-atomistic model. c) RVE consisting of 16 grains.

\subsection{Effect of the GB width}

The two-scale model was tested with different GB widths $(h)$ for both textures (LA and HA), and two grain sizes (3.28 and $6.56 \mathrm{~nm}$ ) on a RVE consisting of 16 grains and 34 GBs. The boundary conditions used for these tensile tests and the RVE are presented in Figure 3. It is found that the RVE mechanical behavior highly depends on the GBs widths. For example, Figure 4 shows the stress-strain curves resulting from GB widths $h$ varying between 0.4 and $1 \mathrm{~nm}$ for all GBs. In the specific case of the LA texture and a mean grain size of $6.56 \mathrm{~nm}, h$ is found to affect the elastic behavior, the limit of elasticity, and the maximal stress that can be sustained before failure. Also, we assert here that, for all textures and grain sizes, decreasing the GB thickness increases the overall stiffness. Consequently, particular attention should be given to the calibration of the GB width in order to properly simulate the mechanical behavior of NC materials.

Unlike the work presented in Ref. [19] where $h$ was arbitrarily set to $1 \mathrm{~nm}$, the models are here calibrated to include each GB width using the QC method. The centrosymmetry parameter $p$, see Ref. [35], is used to detect the crystallographic defects present in the GBs. We identify $h$ when the GB relaxation step required to obtain the best GB energy configuration with QC is over. The threshold chosen for this study is set to $p=0.1$ so that every atom with a p-value lower than this threshold is considered as having a perfect FCC crystal stacking. The determination of $h$ was done by considering only $80 \%$ of the bicrystal interface as this was done for the calculation of the GB energy in Ref. [24], thus ensuring that atoms near free surfaces presenting higher p-values were not counted as part of the $h$ calculation. $h$ was taken as the distance, or the width of the strip of material between the furthest atom from the GB interface of the upper grain and the one of the lower grain. In the LAB cases, the interface is generally considered as an array of dislocations, 


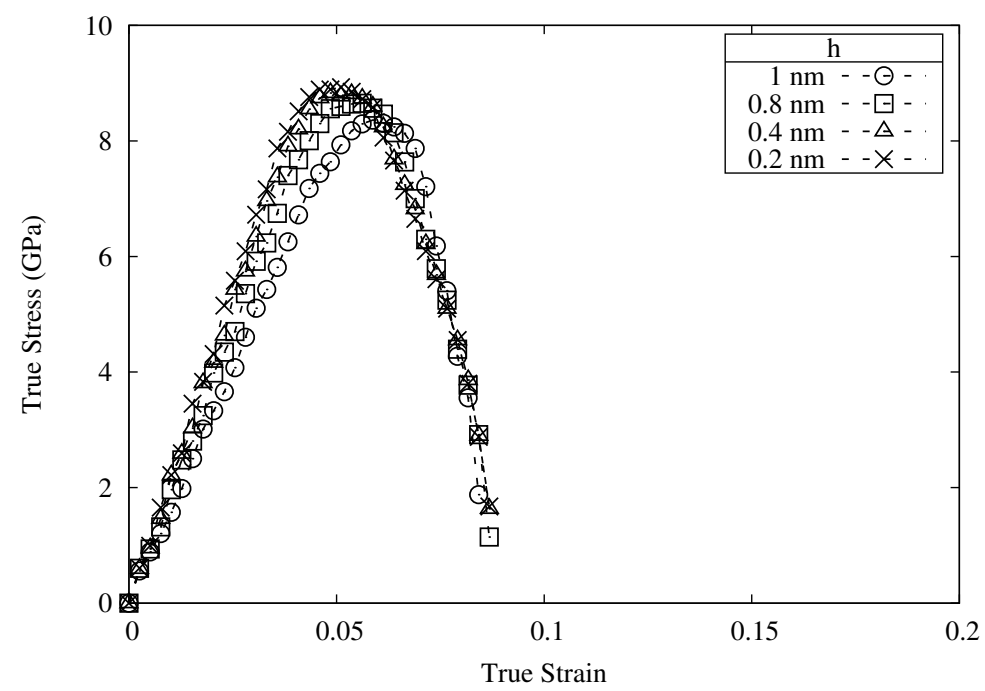

Figure 4: Two-scale model strain-stress curves from tensile tests on the RVE for different GB widths ranging from 0.4 to 1 nm for the LA texture and grain size equal to $6.56 \mathrm{~nm}$.

involving less atoms in the GB structuring than for HABs for which the reorganization is far more random. One can argue that a more accurate determination of the GB width may be based on the number of atoms involved in the reorganization, but this would not fit with the $h$ dimension of the model. By using this method, HABs are found to be wider than LABs, see Figure 5. The obtained GB width is plotted in Figure 6 as a function of the GB misorientation. It is found that HAB widths spread from 12.612 to $16.645 \AA$, while LAB widths never exceed $11.29 \AA$ and always remain above $6.791 \AA$ for the specific misorientations considered.

With a view to facilitating the GB width calibration, we also wanted to assess whether assigning a unique value to $h$ for all the GBs of a given texture would have consequences on the predictions of the mechanical behavior. To this end, tensile loads are applied to the same RVE, see Figure 3. The stress-strain curves are presented in Figure 7. In the HA case and for both grain sizes, no significant discrepancy is observed when $h$ is set to $1.5 \mathrm{~nm}$. Conversely, the LA texture behaves differently when $h$ is set to $0.8 \mathrm{~nm}$ for all LABs. The elastic behavior and the limit of elasticity are the same for both grain sizes but the strain-to-failure is subjected to variations when the grain size is $6.56 \mathrm{~nm}$. The deformed configurations are presented in Figure 8. For the HA texture, full GB width calibration or setting $h$ to $1.5 \mathrm{~nm}$ has no impact on the evolution of the crack propagation, and this results in similar strain-to-failures whatever the calibration method is. The discrepancies that were observed for the LA texture concerning the strain-to-failures echo the different paths being taken by the crack when considering both calibration methods. From this, it is clear that averaging 


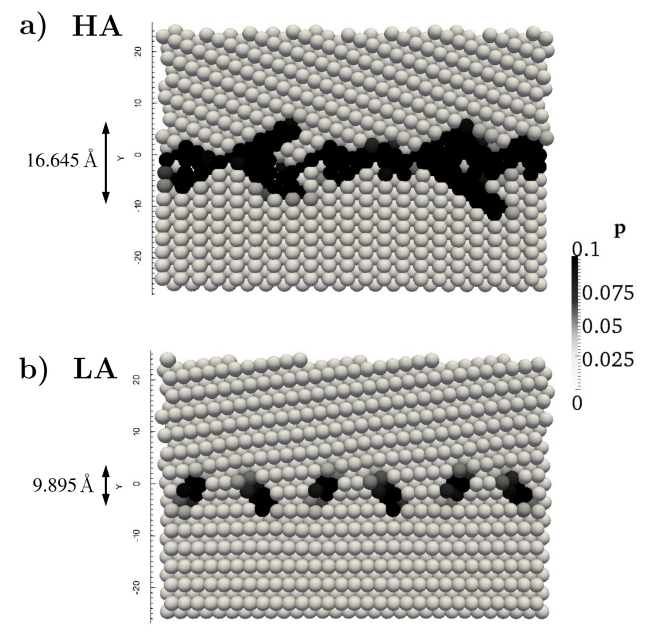

Figure 5: GB width of two representative GB types where $p$ is the centrosymmetry parameter. The same scale for $p$ is used to highlight the width difference. a) HA. b) LA.

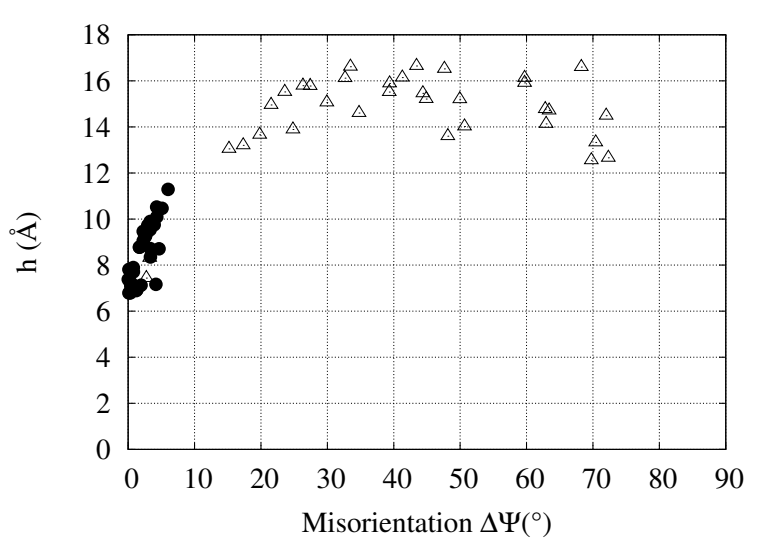

Figure 6: GB width $h$ vs. misorientation $\Delta \Psi$. Triangles symbolise the widths of GBs in the HA texture while the filled circles correspond to the widths of GBs in the LA texture. Two triangles are present amongst LA width values because the HA texture consists also of 2 LABs.

GB width is possible for HABs but not for LABs which are more sensitive to $h$.

\subsection{QC fitting process}

The GB simulations presented in Ref. [19] allow for the identification of overall trends in the GB mechanical properties depending on the degree of GB misorientation, focus on the GB shear modulus $G$, the GB yield stress $\sigma_{0}$, the GB strain-to-failure $\delta_{c}$ and the GB critical stress $\sigma_{c}$. We can also identify trends for $h$ from the results presented in Figure 6. These trends are quite different for each GB type, HA or LA. Within the HA range, it is possible to extract trends from the QC results according to the GB misorientation while results variations are far too important within the LA range, making accurate mechanical behavior predictions of LABs unlikely and consequently in need of individual GB simulations. In order to achieve reproducible results and avoid duplicating work, we have chosen to fit the HABs results for which trends are clearly identified. This fitting process paves the way for more complex HA textures when a large number of grains is present, as it will be the case in the next Section. Fitted curves for all the parameters are presented in Figure 9 and the corresponding equations are reported in Table 2. With regards to the intragranular plasticity and in order to bring together all the parameters needed for larger RVEs simulations, we reproduce here in Figure 9(f) the initial CRSS $g_{0}$ of FCC slip systems as a function of the pseudo grain size $h_{G B}$ as extracted from nanoindentation tests [19]. 

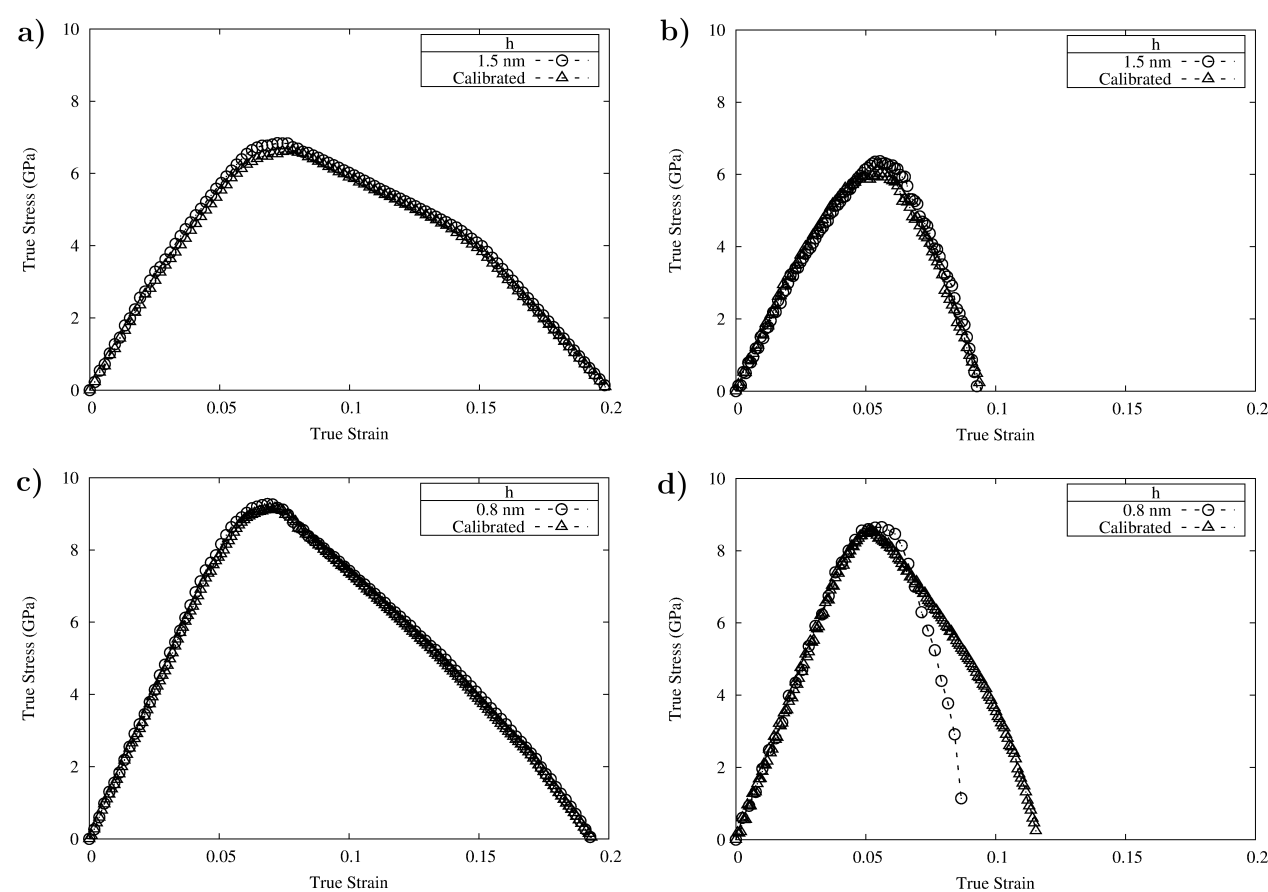

FiguRE 7: Homogeneous width calibration vs. full GB width calibration for both grain textures and sizes: a) HA texture, 3.28 $\mathrm{nm}$; b) HA texture, $6.56 \mathrm{~nm}$; c) LA texture, $3.28 \mathrm{~nm}$; d) LA texture, $6.56 \mathrm{~nm}$.

a)

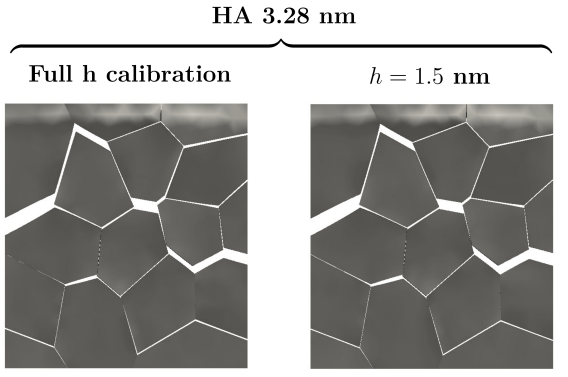

b)

LA $6.56 \mathrm{~nm}$

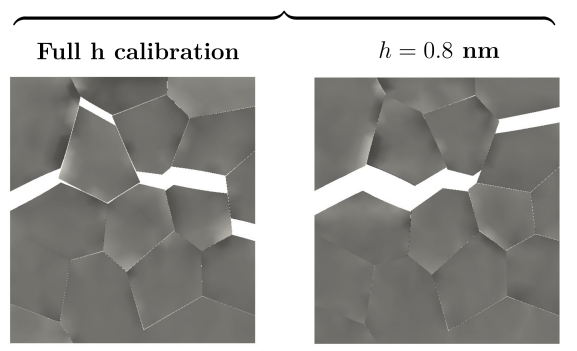

FiguRE 8: Influence of $\mathrm{h}$ on the intergranular crack propagation. a) HA texture with a grain size equal $3.28 \mathrm{~nm}$. b) LA texture with a grain size equal to $6.56 \mathrm{~nm}$. 


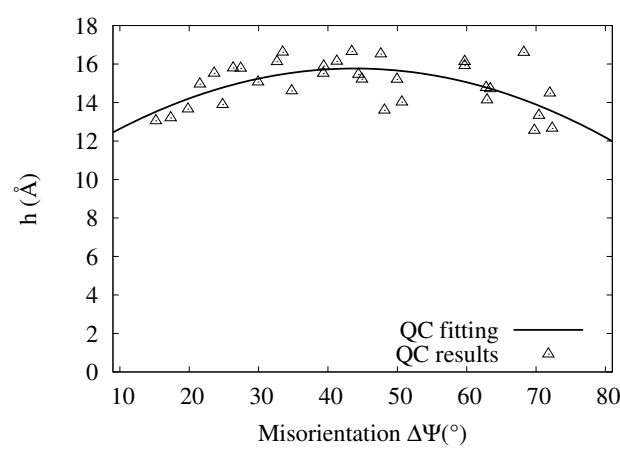

(a)

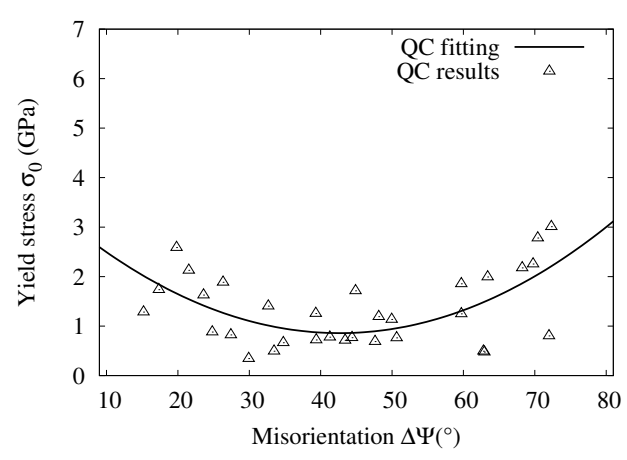

(c)

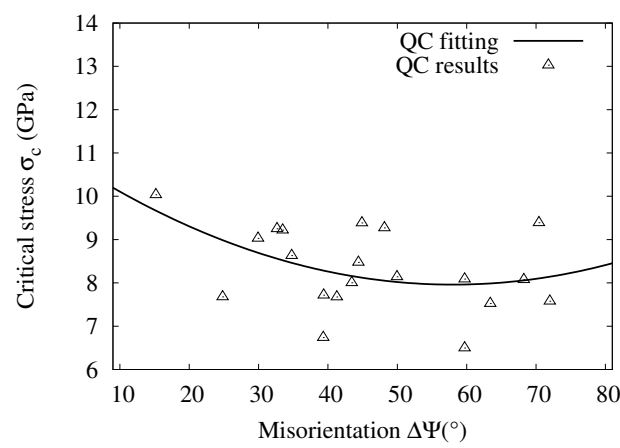

(e)

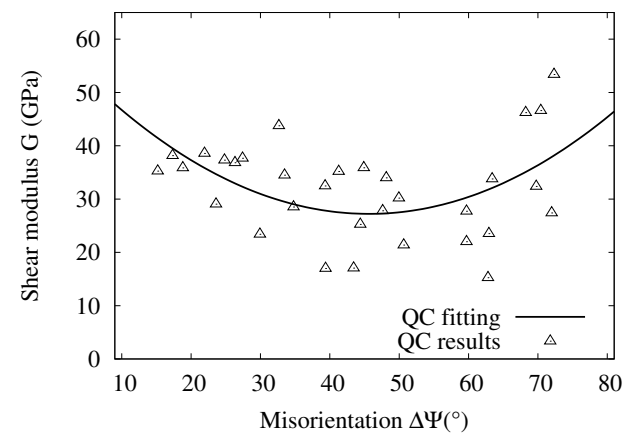

(b)

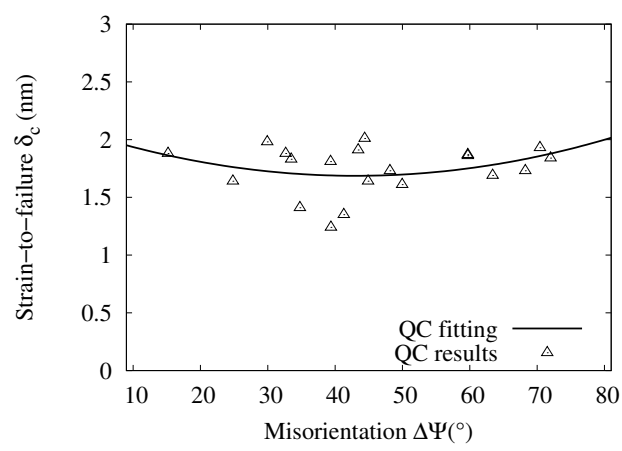

(d)

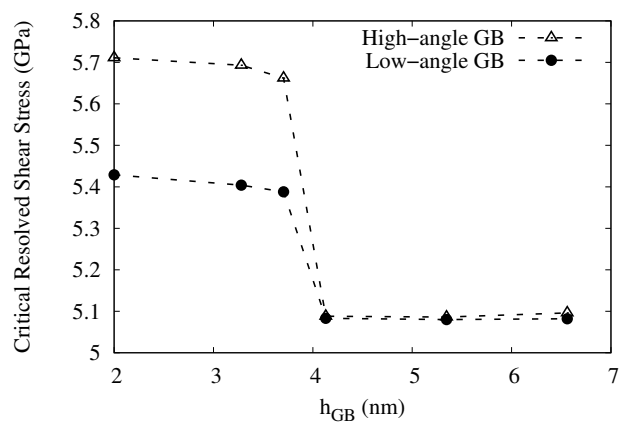

(f)

Figure 9: Fitting of GB parameters: a) $h$, b) $G$, c) $\sigma_{0}$, d) $\delta_{c}$, e) $\sigma_{c}$. f) CRSS $\left(g_{0}\right)$ evolution with $h_{G B}$ for HA and LA GBs. 


\begin{tabular}{|l|c|c|c|}
\hline & $\mathrm{a}$ & $\mathrm{b}$ & $\mathrm{c}$ \\
\hline$h(\AA)$ & -0.00273431 & 0.239748 & 10.5098 \\
\hline$G(\mathrm{GPa})$ & 0.0153508 & -1.40075 & 59.1799 \\
\hline$\sigma_{0}(\mathrm{GPa})$ & 0.00153539 & -0.130957 & 3.65036 \\
\hline$\delta_{c}(\mathrm{~nm})$ & 0.000228623 & -0.0196728 & 2.10974 \\
\hline$\sigma_{c}(\mathrm{GPa})$ & 0.000931509 & -0.108071 & 11.0947 \\
\hline
\end{tabular}

TABLE 2: Fitting values of $\mathrm{a}, \mathrm{b}$ and $\mathrm{c}$ in the polynomial equation $a \Delta \Psi^{2}+b \Delta \Psi+c$ for all GB parameters.

\section{Two-scale model vs. fully-atomistic model}

In this Section, we present the fully-atomistic model that we will compare with the fully calibrated two-scale model of Section 3.

\subsection{Fully-atomistic model}

The two-scale continuum model fully calibrated for $\mathrm{NC}$ copper is compared with the fully-atomistic ones simulated with the QC method. To this end, the fully-atomistic QC model is set for both textures, HA and LA, and the two mean grain sizes 3.28 and $6.56 \mathrm{~nm}$. All simulations consisted of 16 grains, where dimensions depend on the mean grain size: $117 \AA \times 117 \AA \times 2.55619 \AA$ and $233 \AA \times 233 \AA \times 2.55619 \AA$ for a grain size of $3.28 \mathrm{~nm}$ and $6.56 \mathrm{~nm}$, respectively.

The polycrystalline structure was constructed as follows. The GBCD was first created using a Voronoï diagram construction. All atoms were added using the Bravais lattice vectors starting from the 16 reference atoms. A common tilt axis along the [1]0] direction was assigned to each grain and in-plane misorientations were set according to the texture type. Periodic boundary conditions were applied along the out-of-plane direction in the entire model. Consequently, this work focuses on 2-D columnar-grained microstructures, and as such, plastic deformation may be different to those that could be observed for 3-D polycrystalline structures whereas this assertion is valid for all QC simulations presented in this study, the results presented here can be extended to 3-D and are expected to be qualitatively similar in the plane-strain state.

The total energy was minimized using the conjuguate gradient method until the addition of out-ofbalance forces was found to be lower than $10^{-3} \mathrm{eV} . \AA^{-1}$. All structures were relaxed under zero pressure to obtain the lowest state of energy. During this relaxation step, all atoms at the bottom of the sample were fixed in all directions while those on the left and the right sides were fixed in the $X$ - and $Z$-directions, see Figure 3a. These strong boundary conditions were set up so as to avoid the complete crystallographic reorganization of NCs, especially in the case of the LA type where GBs can disappear after relaxation. Atoms at the top of the samples were then subjected to tensile loadings, see Figure 3b, by means of incremental 


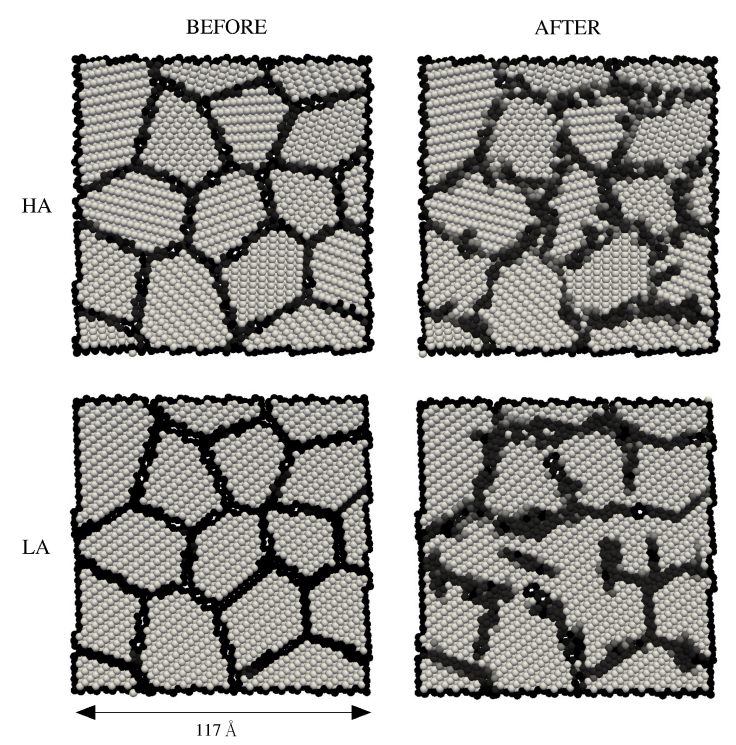

FiguRE 10: Snapshots for both HA and LA textures for a mean grain size of $3.28 \mathrm{~nm}$, before and after the relaxation step.
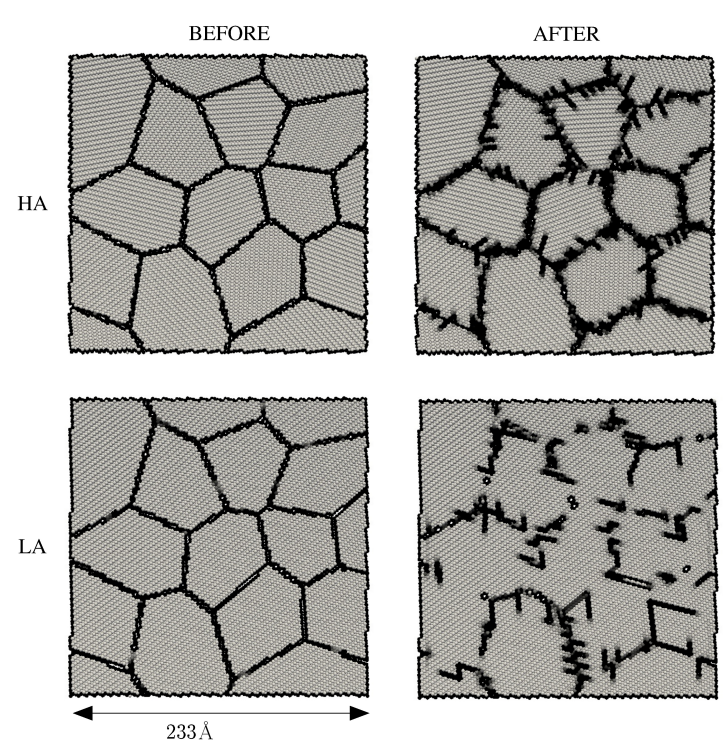

Figure 11: Snapshots for both HA and LA textures for a mean grain size of $6.56 \mathrm{~nm}$, before and after the relaxation step.

displacements of $0.25 \AA$ in the Y-direction until the sample reached a deformation of $20 \%$. Between each loading step a new energy minimization was performed. Moreover, the centrosymmetry parameter $p$ [35] was computed at each loading step to allow for the detection of planar defects during deformation. Both HA and LA textures, with mean grain sizes equal to 3.28 and $6.56 \mathrm{~nm}$, are presented with this $p$ value in Figures 10 and 11, respectively. Although the relaxation step implies a slight distortion in the grain shape, it appears that GBCDs retain overall their HA or LA character.

It is worth noting that the QC method was originally conceived to model atomistic systems without explicitly treating every atom in the problem by judiciously eliminating unnecessary degrees of freedom. In doing so, the QC method reduces calculations time while keeping an atomistic description where needed. In this work, this aspect of QC was not used for the fully-atomistic simulations as the small size of the grains require all the atoms to be modelled. Therefore, no degree of freedom was eliminated which implies a significant slowdown of QC.

\subsection{Results}

For both models, the yield stresses were determined as being the stresses at which the residual plastic strain reaches $0.2 \%$. Figure 12 shows the simulated stress-strain curves of both models NC simulations for both HA and LA textures and for both grain sizes with the same loading conditions. 
In the QC models, with both textures, the simulation cells initially deform elastically until reaching a critical level of stress. In the HA texture case, yield stresses reach 8.49 GPa and 8.79 GPa for a mean grain size of $3.28 \mathrm{~nm}$ and $6.56 \mathrm{~nm}$, respectively, while $9.78 \mathrm{GPa}$ and $9.89 \mathrm{GPa}$ are reached in the LA cases, for a mean grain size of $3.28 \mathrm{~nm}$ and $6.56 \mathrm{~nm}$, respectively. For each texture, it appears that the smaller the grain size the sooner the yield point, corresponding to the material softening when the grain size decreases, and thus showing the ability of the fully-atomistic QC model to capture the reverse Hall-Petch (RHP) effect.

Similar observations can be made when considering the two-scale model results, where textures with small grains deviate faster from the elastic regime in agreement with Ref. [9]. For a grain size of $6.56 \mathrm{~nm}$, yield stresses in $\mathrm{HAB}$ and $\mathrm{LAB}$ textures are $5.55 \mathrm{GPa}$ and $8.11 \mathrm{GPa}$, respectively, and decrease to 4.26 GPa and $7.93 \mathrm{GPa}$ for a grain size of $3.28 \mathrm{~nm}$. It is worth noting that these yield stresses are higher than those observed in a recent work [19] for which boundary conditions were less constrained. Additionally, yield stresses in the HAB textures are always lower than in the LAB ones which is true for both models.

When comparing stress-strain curves obtained with both models, a good agreement is achieved qualitatively for different textures and grain sizes. However, the two-scale model is associated in each case with yield stresses lower than those observed in the fully-atomistic model, due to the role of triple junctions, i.e. the junction between three grains, which are not taken into account in the two-scale model. These triple junctions, absent in the GB decohesion calibration of the two-scale model, seem to be responsible for a larger GB stiffness by reducing the GB motion freedom. Therefore, our two-scale model may underestimate the GB stiffness in opening, and thus underestimate the global stiffness of the NC model. This hypothesis is also supported by the decrease of the NC stiffnesses in both textures when the grain size decreases from $6.56 \mathrm{~nm}$ to $3.28 \mathrm{~nm}$ in the two-scale model, see Figures 12a and c. Indeed, the effect of the absence of the triple junction calibration in the two-scale model seems even more important when the grains are smaller. In that case, the GBs lengths involved are smaller which implies a higher proportion of triple junctions in the $\mathrm{NC}$ and consequently, an apparently softer $\mathrm{NC}$ material.

Deformed configurations for both models are presented in Figure 13 at the yield point and at $\epsilon=10 \%$. At yield point, remarkably, a very good agreement is observed in all cases and the crack initiation occurs at the same GBs if we compare both models. For 10\% deformation, the GB networks responsible for the failure of the RVEs remain closely connected. We therefore demonstrate here that the two-scale model makes it possible to predict accurately the weakest links and crack initiation loci of these GB networks.

Regarding the calculation time, clear benefits are found when using the two-scale model. For instance, the computation time required to fully calibrate the two-scale model of a complete HA texture for an average 

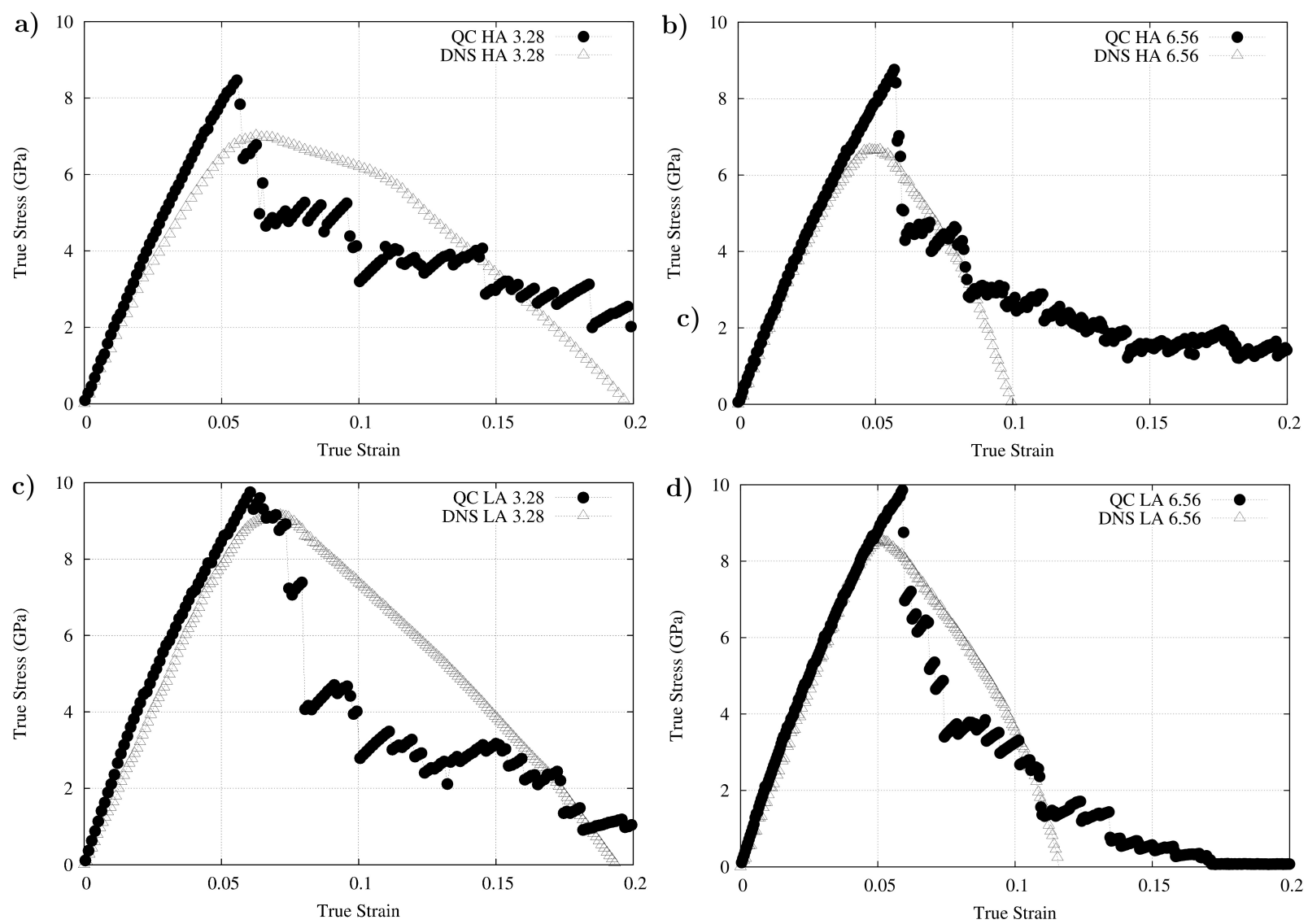

FiguRE 12: Comparison of the stress-strain curves of both models. a) HA texture for a $3.28 \mathrm{~nm}$ mean grain size. b) HA texture, $6.56 \mathrm{~nm}$. c) LA texture, $3.28 \mathrm{~nm}$. d) LA texture, $6.56 \mathrm{~nm}$. 
grain size of $6.56 \mathrm{~nm}$ is about 89 hours with a $3.33 \mathrm{GHz} \mathrm{CPU}$, to which must be added the time required to apply tensile load on this RVE (7451 tetrahedral elements), i.e. 15 hours and 30 minutes, totalling 104 hours and 30 minutes. On the other hand, the fully-atomistic QC model requires about 95 hours with the same device to compute the same texture. For this specific RVE size, both methods are roughly equivalent but the interest of using such a two-scale method appears obvious for larger structures as in next Section, when studying different loading with the same structure, or when using Figure 9 in the HA case, instead of repeating all the bicrystal simulations.

\section{Dogbone study}

\subsection{Dogbone description}

To illustrate the efficiency of the two-scale method when dealing with a large number of grains, tensile tests were performed on two large dogbones presenting mean grain sizes of $3.28 \mathrm{~nm}$ ("A") and $6.56 \mathrm{~nm}$ ("B"). The two structures consisted of 103 grains and 251 GBs. The misorientation distribution is presented in Figure 14. For this texture, only one GB belonged to the LA type and all the other ones belonged to the HA type, thus giving a proportion of $99.6 \%$ for the HABs in the texture. Due to the difficulty of fitting the LAB features, the QC calibration was performed for this misorientation. However, the mechanical responses of all the HABs were calibrated using the fitting parameters in Figure 9. Both meshes contained 2,201 nodes for 8,934 tetrahedral and 5,458 nodes for 21,162 tetrahedral elements, respectively. Dimensions and tensile boundary conditions are presented in Figure 15.

\subsection{Dogbone results}

The stress-strain curves achieved for the tensile tests are presented in Figure 16. According to these curves, we see that if we consider the whole deformation of each dogbone, the elastic part takes a large place in the case B (larger grains) whereas the plastic part is more pronounced in the case A (smaller grains). Moreover, A appears to be less rigid than B. As a result, B fails for smaller strain than A. These last assertions confirm the ability of the model to capture the RHP effect. Snapshots presented in Figure 17 represent the deformed configurations of both dogbones A and B for three different steps: step 1 is taken during elastic deformation, step 2 is taken in the plastic part of the deformation and finally, step 3 corresponds to the maximum strength experienced by the dogbones. We have chosen to show within the grains $g-g_{0}$, where $g_{0}$ and $g$ are the initial and current CRSS, respectively, as intragranular plasticity is known to be absent when this variable is equal to zero. GB sliding is significantly more important in case A 
YIELD POINT

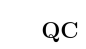

a) HA $3.28 \mathrm{~nm}$

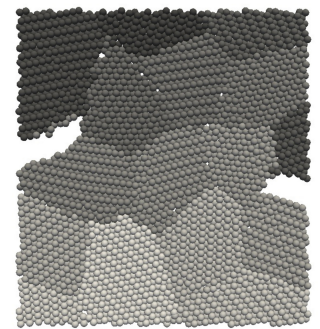

b) LA $3.28 \mathrm{~nm}$

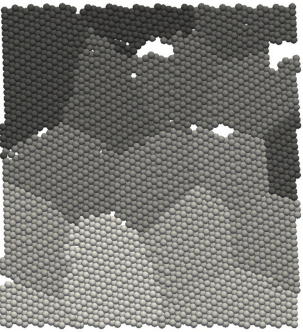

c) HA $6.56 \mathrm{~nm}$

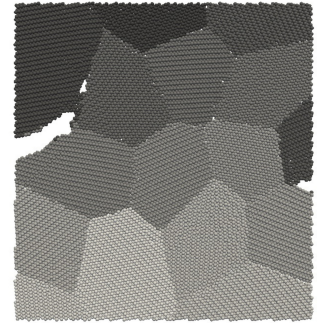

d) LA $6.56 \mathrm{~nm}$

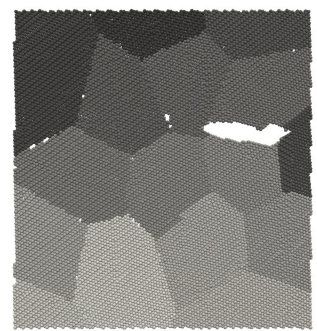

2 SCALE-MODEL
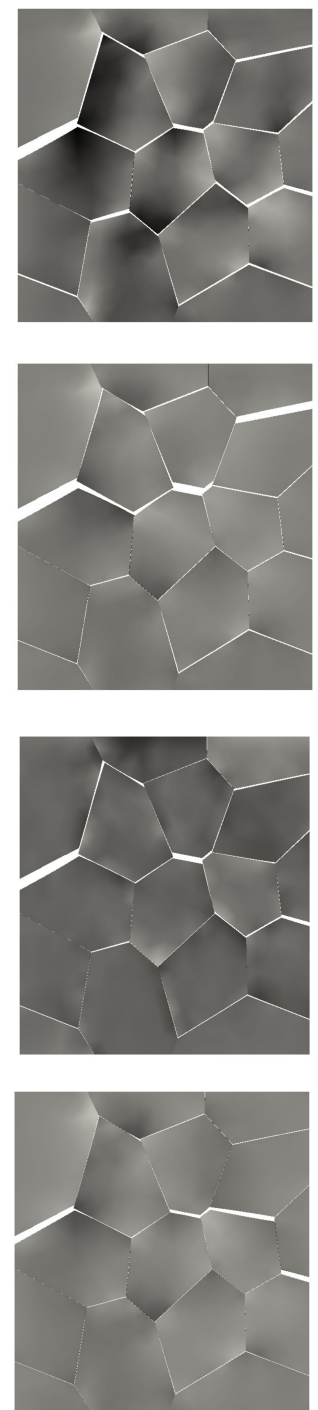

$\epsilon=10 \%$

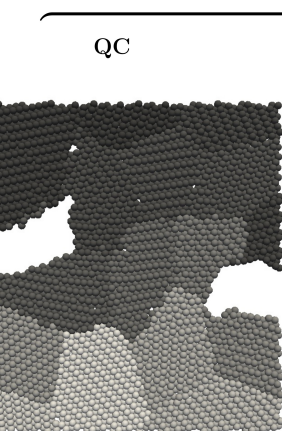

2 SCALE-MODEL
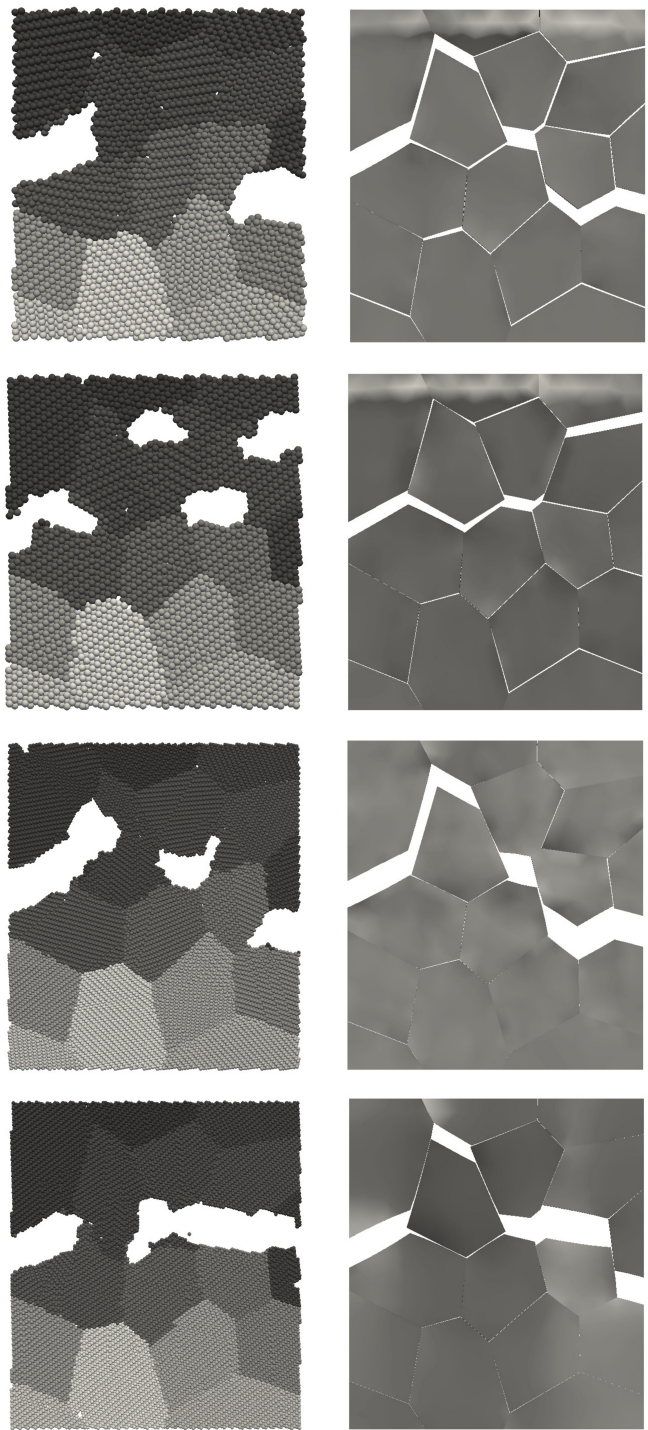

Figure 13: Deformed configurations for both models at yield point and for a $\epsilon=10 \%$ deformation. 


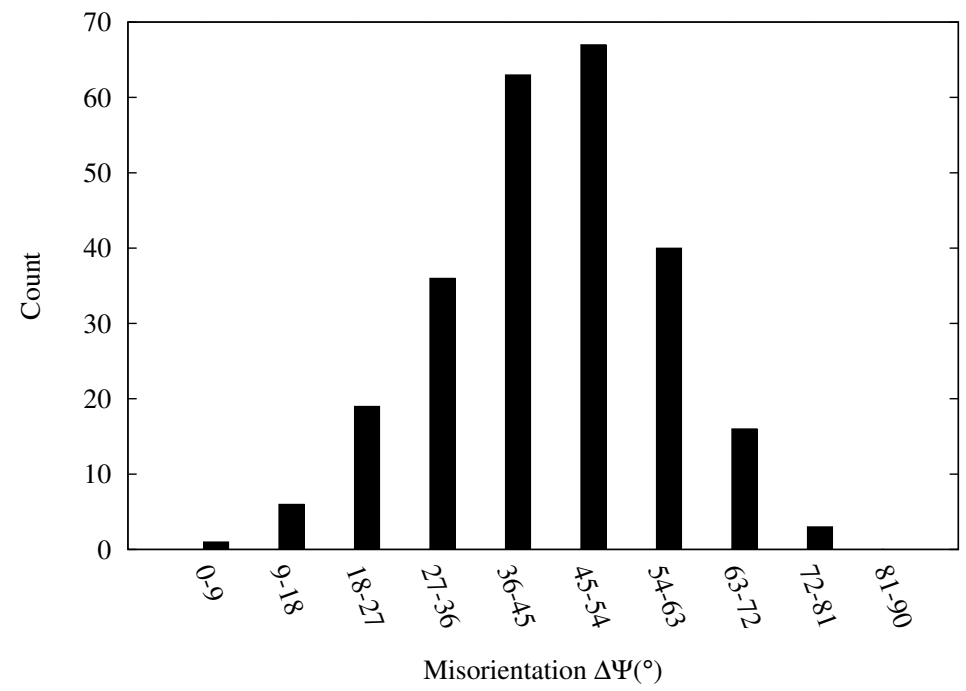

FiguRE 14: Misorientations distribution of the dogbone.

a)

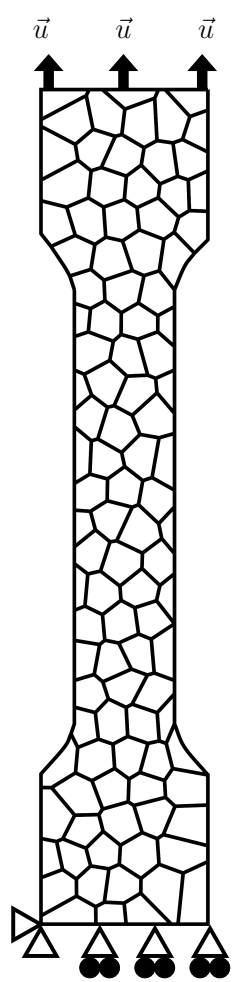

b)

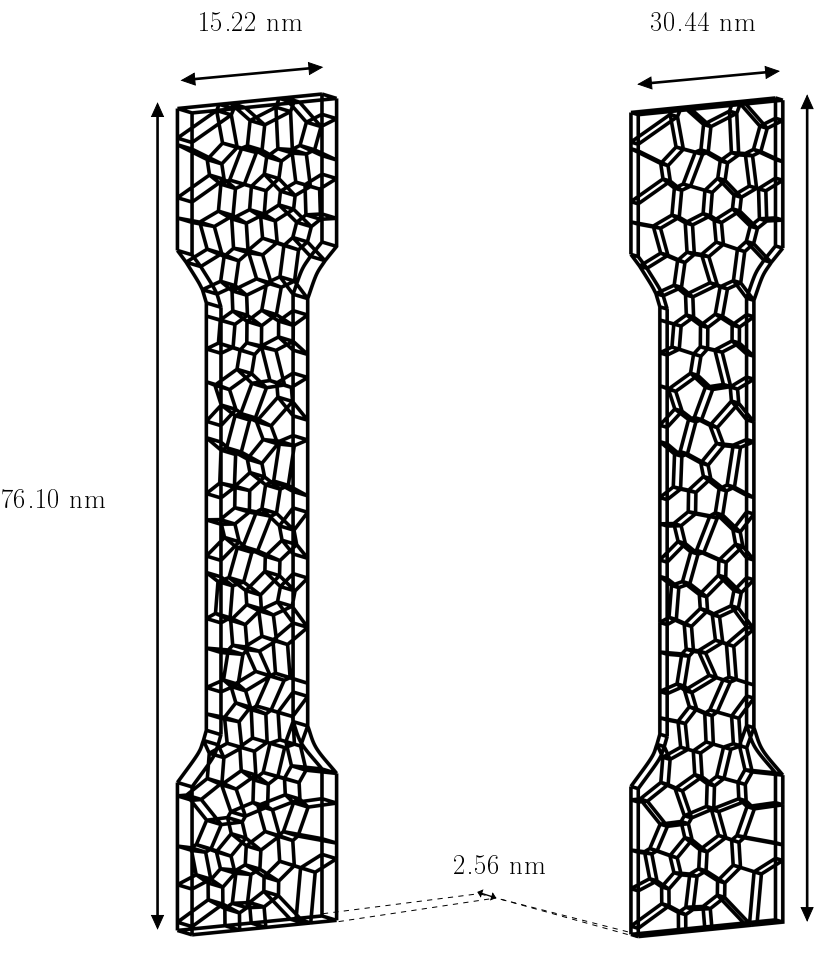

$152.21 \mathrm{~nm}$

FIGURE 15: Boundary conditions and dogbone dimensions. a) Tensile loading boundary conditions. Nodes are fixed in the Z directions. Dimensions of dogbones with a grain size set to b) $3.28 \mathrm{~nm}$ and c) $6.56 \mathrm{~nm}$. 


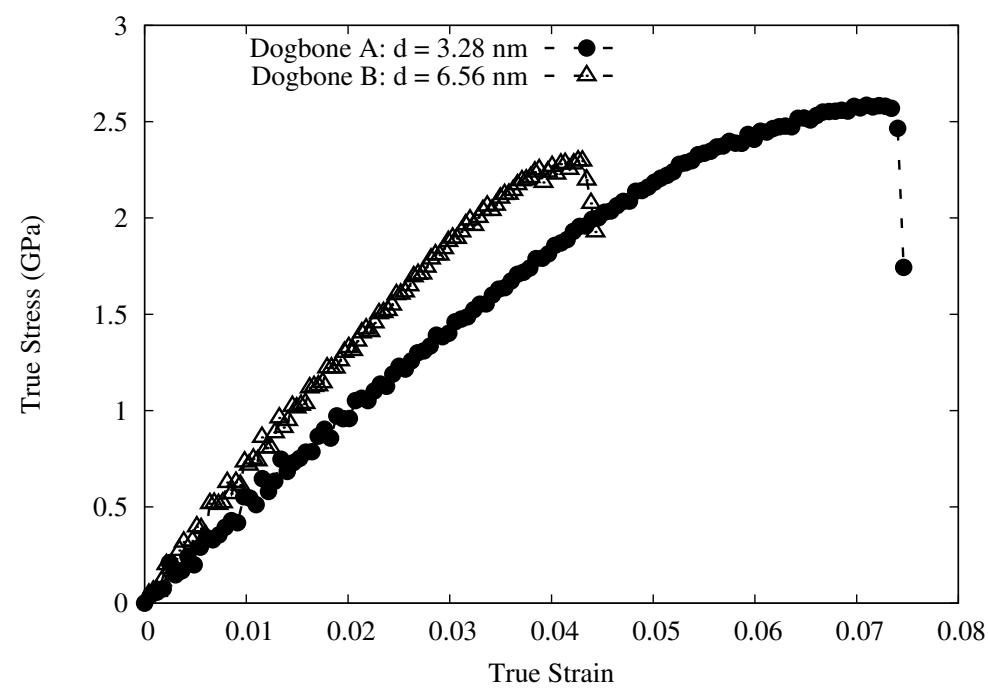

FigurE 16: Stress-strain curves results for the dogbones A (smaller grains) and B (larger grains).

as shown in step 3, coupled to the high proportion of intergranular plasticity in the deformation process of A. In contrast, comparing A step 2 and B step 3 shows that even for a lower strain in B (4.26\% versus $5.01 \%$ for A) $g-g_{0}$ is overall higher in B. In fact, while intragranular plasticity seems to be more homogeneous in grains of A, many black spots on B indicate a more pronounced intragranular activity. This highlights the higher intragranular plasticity occuring in B. In summary, the two-scale model confirms that the failure mechanism in NC dogbones is more predominantly intragranular for large grain sizes and intergranular at smaller grain sizes.

\section{Conclusion}

In this work, a two-scale model atomistically-informed at the lowest scale has been proposed. The calibration of the GBs and bulk crystals was done by means of GB sliding and opening; and nanoindentation QC simulations, respectively, enabling the creation of a climate of competition between intergranular and intragranular plasticity.

The observation of the relaxed [1ํㅣ ] tilt GBs resulting from QC simulations allowed for the obtention of the GB width distribution as a function of the GB misorientation. It is concluded that HABs are wider than LABs. Moreover, the simulations of RVEs consisting of 16 grains exhibited an almost identical behavior at the polycrystalline level when using average width of $1.5 \mathrm{~nm}$ for all HABs. In turn, LABs were found to be more sensitive to width calibration and still require to be calibrated individually in a polycrystalline 


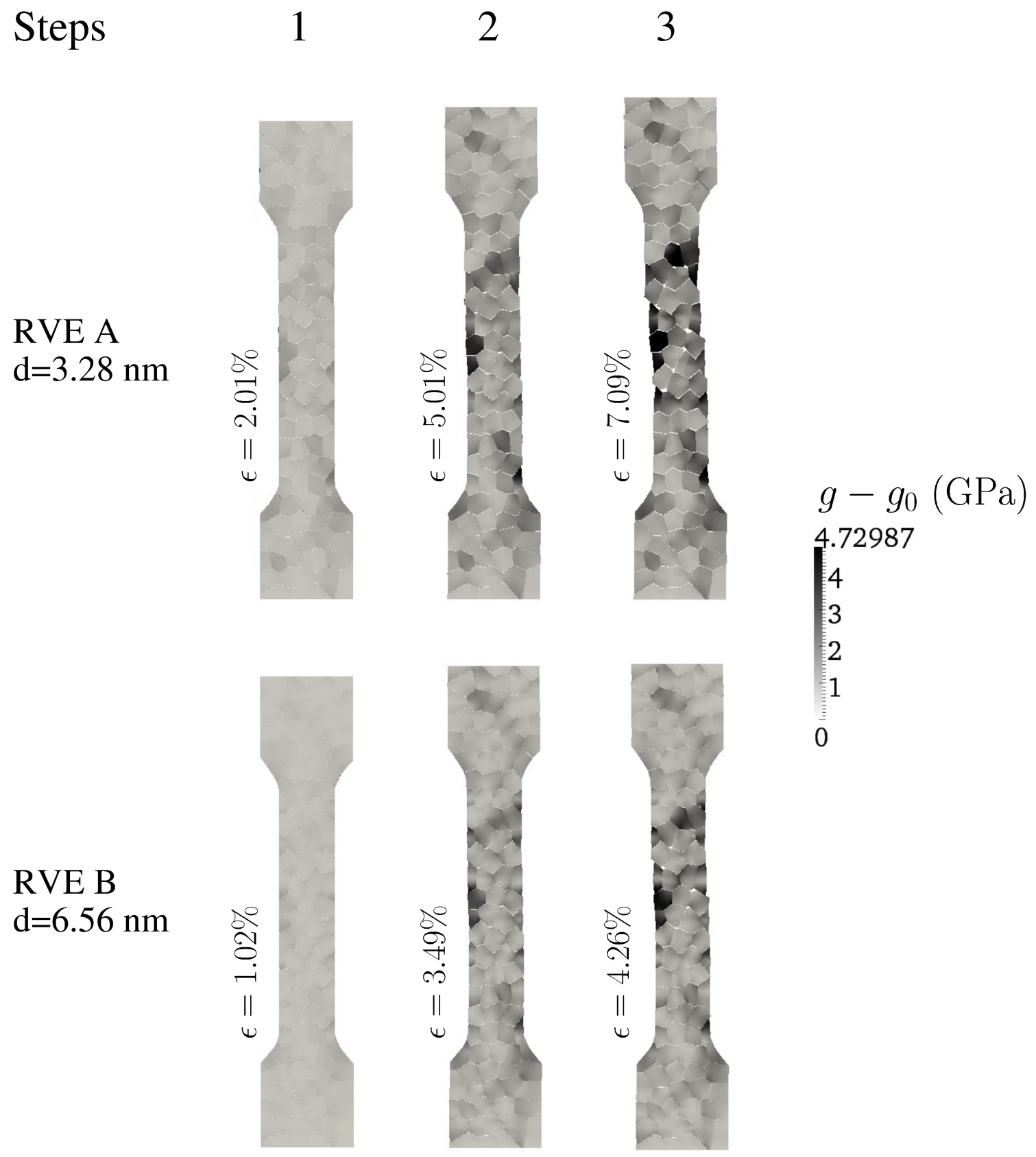

Figure 17: Deformed configurations of dogbones A and B. Three steps of deformation are considered: step 1 is elastic, step 2 is plastic and step 3 is at maximum strength. 
aggregate.

Built up on previous work [19], mechanical properties evolution equations as a function of GB misorientation have been proposed for HABs, thus avoiding future QC simulations and significantly improving the calculation time of future continuum simulations.

The prediction of the evolution of the crack propagation in both two-scale and fully-atomistic models is similar. However, yield stresses measured with the two-scale model are found systematically smaller than in QC simulations. This discrepancy is believed to be due to the absence of triple junctions considerations when calibrating the GB opening with QC, thus underestimating the GB critical stresses $\sigma_{c}$. The smaller the grain size, the larger the proportion of triple junctions, and the softer the structures predicted by two-scale model. These triple junctions $[36,37]$ are also already known to play a major role in the propagation of intergranular cracks and are recognized as being able to nucleate, block, deviate or transmit them [38]. Besides, special mechanisms such as GB migration can be responsible for the growth of a new grain during straining [39] at triple junctions. Concentrations of stresses at the triple junctions observed in the two-scale model cause the appearance of intragranular plasticity, surely increased, but that does not allow to reflect the specific nano mechanisms involved in this area. Thus and as for the GBs, the particular mechanisms taking place in the triple junctions may also be considered by adding special elements calibrated with atomistic simulations in order to improve the model.

It must finally be emphasized that the two-scale model stresses remain overvalued compared to dynamics simulations as noticed in Ref. [19]. This discrepancy arises from the 2-D nature of the QC method and from the fact that no thermally activated processes are accounted for in the simulations.

The results are also overvalued compared to experiments where nanoscale voids are present and using the void-induced stress model recently presented in Ref. [40] would improve the mechanical calibration of GBs.

\section{Acknowledgments}

F.S. gratefully acknowledges support from the National Science Foundation (grant number DMR-0747658).

\section{References}

[1] M. Dao, L. Lu, R. J. Asaro, J. T. M. De Hosson, E. Ma, Acta Materialia 55 (2007) 4041-66.

[2] M. Meyers, A. Mishra, D. Benson, Progress in Materials Science 51 (2006) 427-556.

[3] K. S. Kumar, H. Van Swygenhoven, S. Suresh, Acta Materialia 51 (2003) 5743.

[4] C. J. Youngdahl, P. G. Sanders, J. A. Eastman, J. R. Weertman, Scripta Materialia 37 (1997) 809.

[5] K. W. Jacobsen, J. Schiøtz, Nature Materials journal 1 (2002) 15. 
[6] J. Schiøtz, K. W. Jacobsen, Science 301 (2003).

[7] J. Li, A. K. Soh, Modelling and Simulation in Materials Science and Engineering 20 (2012).

[8] J. R. Weertman, D. Farkas, K. Hemker, H. Kung, M. Mayo, R. Mitra, H. Van Swygenhoven, MRS Bulletin 24 (1999) $44-53$

[9] A. Jérusalem, L. Stainier, R. Radovitzky, Philosophical Magazine 87 (2007) 2541-2559.

[10] H. Van Swygenhoven, P. M. Derlet, A. G. Froseth, Nature Materials 3 (2004) 399-403.

[11] D. Wolf, V. Yamakov, S. Phillpot, A. Mukherjee, H. Gleiter, Acta Materialia 53 (2005) 1-40.

[12] F. Sansoz, V. Dupont, Applied Physics Letters 89 (2006).

[13] V. Dupont, F. Sansoz, Acta Materialia 56 (2008) 6013-6026.

[14] F. Sansoz, K. D. Stevenson, Physical Review B 83 (2011) 224101-1 224101-9.

[15] Y. J. Wei, L. Su, C. Anand, Acta Materialia 54 (2006) 3177-3190.

[16] H. H. Fu, D. J. Benson, M. A. Meyers, Acta Materialia 52 (2004) 4413-4425.

[17] D. Warner, F. Sansoz, J. Molinari, International Journal of Plasticity 22 (2006) 754.

[18] Y. Wei, L. Anand, Journal of Mechanics and Physics of Solids 52 (2004) 2587-2616.

[19] V. Péron-Lührs, A. Jérusalem, F. Sansoz, L. Stainier, L. Noels, Journal of Mechanics and Physics of Solids 61 (2013) $1895-1914$.

[20] E. B. Tadmor, M. Ortiz, R. Phillips, Philos Mag A 73 (1996) 1529-1563.

[21] V. B. Shenoy, R. Miller, E. B. Tadmor, D. Rodney, R. Phillips, M. Ortiz, Journal of Mechanics and Physics of Solids 47 (1999) 611-642.

[22] S. N. Kuchnicki, A. M. Cuitiño, R. A. Radovitzky, International Journal of Plasticity 36 (2006) 1.

[23] A. M. Cuitiño, M. Ortiz, Modelling and Simulation in Materials Science and Engineering 1 (1993) $225-263$.

[24] F. Sansoz, J. F. Molinari, Scripta Materialia 50 (2004) 1283-1288.

[25] F. Sansoz, J. F. Molinari, Acta Materialia 53 (2005) 1931-1944.

[26] Z. Zhao, S. N. Kuchnicki, A. M. Radovitzky, R. A. Cuitiño, Acta Materialia 55 (2007) 2361-2373.

[27] M. Ortiz, A. Pandolfi, International Journal for Numerical Methods in Engineering 44 (1999) 1267.

[28] G. J. Thomas, R. W. Siegel, J. A. Eastman, Scripta Metallurgica and Materialia 24 (1990) $201-209$.

[29] H. Kung, P. G. Sanders, J. R. Weertman, Advanced Materials for the twenty-first Century (1999) $455-463$.

[30] M. Ortiz, E. P. Popov, Computer Methods in Applied Mechanics and Engineering 90 (1982).

[31] E. B. Tadmor, R. Miller, R. Phillips, Journal of Materials Research 14 (1999) 2233.

[32] D. M. Sailor, A. Morawiec, B. I. Adams, G. S. Rohrer, Interface Science 8 (2000) 131-140.

[33] L. N. Brewer, M. A. Othon, L. M. Young, T. M. Angeliu, Microscopy and Microanalysis 12 (2006) 85-91.

[34] N. Shigematsu, D. J. Prior, J. Wheeler, Journal of Microscopy 224 (2006) 306-321.

[35] C. L. Kelchner, S. J. Plimpton, J. C. Hamilton, Physical Review B 60 (1998) 11085-11088.

[36] C. A. Schuh, M. Kumar, W. E. King, Acta Materialia 51 (2003) 687-700.

[37] M. Kumar, W. E. King, A. J. Schwartz, Acta Materialia 48 (2000) 2081-2091.

[38] O. K. Johnson, C. A. Schuh, Acta Materialia 61 (2013) 2863-2873.

[39] A. J. Cao, Y. G. Wei, Physical Review B 76 (2007) 024113.

[40] V. Péron-Lührs, F. Sansoz, L. Noels, Acta Materialia 64 (2014) 419-428. 